\title{
Interpretation and Construction of "Meaning-Senses" within Cross-cultural Context-Extension of Brand Name Translation Theory
}

\author{
Jiafeng Liu ${ }^{1}$ \\ ${ }^{1}$ School of Foreign Languages, Southwest Minzu University, Chengdu, China \\ Correspondence: Jiafeng Liu, School of Foreign Languages, Southwest Minzu University, Chengdu, China. \\ E-mail: 378411817@qq.com
}

Received: September 5, 2017 Accepted: September 24, 2017 Online Published: November 2, 2017

doi:10.5539/ells.v7n4p79 URL: http://doi.org/10.5539/ells.v7n4p79

\begin{abstract}
Integral study of brand name translation needs promoting theoretical category system at relevant macro-meso-micro contextual levels. Taken E-C brand name translation as example, this paper establishes an explanatory frame of generation and construction of "meaning-senses" of translated brand name through discussing the macro-meso-micro contexts and their major factors in the process of brand name translation. This research shows that "named meaning", determined by meso brand contextual factors, should be jointly delivered by micro linguistic contextual factors complying with demands of macro cultural contexts. This research will provide a feasible research dimension for systematic integration of theoretical categorization of pragmatic translation.
\end{abstract}

Keywords: brand name, translation, meaning-senses, generation mechanism; formulation principle

\section{Introduction}

Brand name translation belongs to pragmatic translation. Translated brand names should be closely related to brand image and brand communication system, delivering relevant product information satisfying demands of target consumers. The translation focus should be on "pragmatic meaning" determined by source text context and target text context, of which brand name is dependent, to achieve equivalent pragmatic effect. He Ziran (1992) advanced that to achieve equivalent pragmatic effect, on one hand, one has to pay attention to the "intended meaning" of source text, considering source text's meaning in contexts from the perspective of source text; on the other hand, one has to pay attention to target text context, choosing appropriate linguistic forms of target text to express the intention or intended meaning of source text. The questions needed to be discussed include: what are the connotations of source text context and target text context, of which brand name translation is dependent on; what major contextual factors are involved; how these contextual factors influence or constrain the generation of "meaning-senses" in brand name translation. However, relevant systematic discussion has not been conducted in both domestic and overseas translation fields. Taking E-C brand name translation as example, this paper tries to clarify the connotations of source text context and target text context brand name translation relies on. Discussing the main contextual factors included in source text context and target text context, this paper will systematically analyze the generation mechanism and formulation principles of "meaning-senses" in brand name translation, developing deeply research dimension for translation theory and practice of specific applied texts from macro-meso-micro levels.

\section{Source Text Context and Target Text Context Brand Name Translation Relies on}

\subsection{Connotation and Classification of Context}

Malinowski, an anthropologist, first proposed the term of "context" in 1923 and classified contexts into "linguistic context" and "situational context". Eggins (1994) developed contexts into discourse context, context of situation and context of culture. Wang Dechun (1964) divided contexts into subjective context and objective context. Pragmatist Verschueren (1999) noted that relevant linguistic or non-linguistic constituents in the process of language working were the contexts producing discourse, and the contexts can be divided into cognitive context, communicative context and linguistic context. These classifications of contexts are basically extensive and obscure in definition, and essentially are discussions on the connotation of context at macro-meso-micro 
levels. Discourse (linguistic) context involved in text is micro context, referring to the information content in discourse. Situational context involved in text is meso context, referring to correlative context of situation. Cultural context involved in text is macro context, referring to the specific background a discourse involves.

\subsection{Source Text Context and Target Text Context Brand Name Translation Relies on}

American anthropologist Shaw (1987), from the perspective of translation process, pointed that components of translation context included source text context, target text context and translator. Translator, as the one transferring information of source text and sending information of target text, mainly focuses on appropriate understanding of source text and proper expression of target text. During understanding process, translator fully considers specific contextual factors of source text to find out the optimal correlation between source text and context, thoroughly understanding the connotation and denotation of words of source text in context. In target text, translator not only has to reproduce the situation in source text but also has to consider comprehensively the linguistic context of target text. Therefore, linguistic expressions of target text can be accordant with habits of target language while conforming to social and cultural context, ensuring target readers can understand the content and thoughts of source text precisely.

Brand name is a complex symbol, and a sound and (or) practical meaning represented by the symbol, possessing a series of brand associations or brand attributes constructed in the symbol with enterprise's long efforts and indicating demands of target consumers and brand marketing strategies. It also conveys core associative meaning of brand and reflects the specific cultural background of target market embodied in brand. However, when it turns into target market of target language, the complexity of commodity economy phenomena makes brand name translation beyond linguistic arena and up to cultural psychology and market repositioning. Under the new market environment, target consumers' cultural background, purchasing habit and mode of language use would be different from those of original target consumers, leading to corresponding changes in brand marketing strategies and market positioning of enterprises. Huang \& Chan (2002, p. 68) summarized brand naming principles into market, legal and linguistic elements from the perspective of linguistics. Market elements cover part of macro social and cultural factors and meso branding factors. Legal elements are macro social and cultural factors. And linguistic elements are micro linguistic contextual factors. These contextual factors at different levels together influence the generation and formulation of a brand name translation's "meaning".

In brand name translation, to achieve correct understanding of original brand name's connotation and proper utterance of brand name translation, a translator has to consider macro-meso-micro contexts of both source language and target language, on which brand name translation relies. Macro context of source language refers to social and cultural background a brand name relies on, mainly including political, economic, cultural, historical and legal elements of target market. Meso context refers to branding around brand name made by enterprise, mainly including key components of enterprise's brand building in target market, such as product, target consumers, brand marketing strategy, core brand associations and brand logo. Micro context refers to linguistic environment a brand relies on, mainly including pronunciation, morphological form and "meaning-senses" of a brand name. Correspondingly, macro context of target translation is the macro social and cultural background a brand name translation relies on, consisting of political, economic, cultural, historical and legal elements of target market of target language. Meso context of target text refers to branding made by enterprise around the translated brand name, mainly including components of enterprise's brand building in target market of target language, such as product, target consumers, brand marketing strategy, core brand associations and brand logo. Micro context of target text is the linguistic environment a brand name translation relies on, mainly including linguistic naming standards of a brand name in target language such as ideal phonetic, semantic and morphological features of a brand name in target language.

\section{Generation and Formulation of "Meaning" in Brand Name Translation (Figure 1)}

During the translation process, the meaning of words usually is determined by contexts. It is also applicable to brand name translation. The generation of "meaning" of a brand name translation is not only influenced by macro-meso-micro contexts of original brand name but also restricted by macro-meso-micro contexts of target market of target language. The formulation of a brand name translation's "pronunciation, morphological form and meaning-senses" is affected and constrained by the proportion of contextual factors at different levels. Here is the figure. 


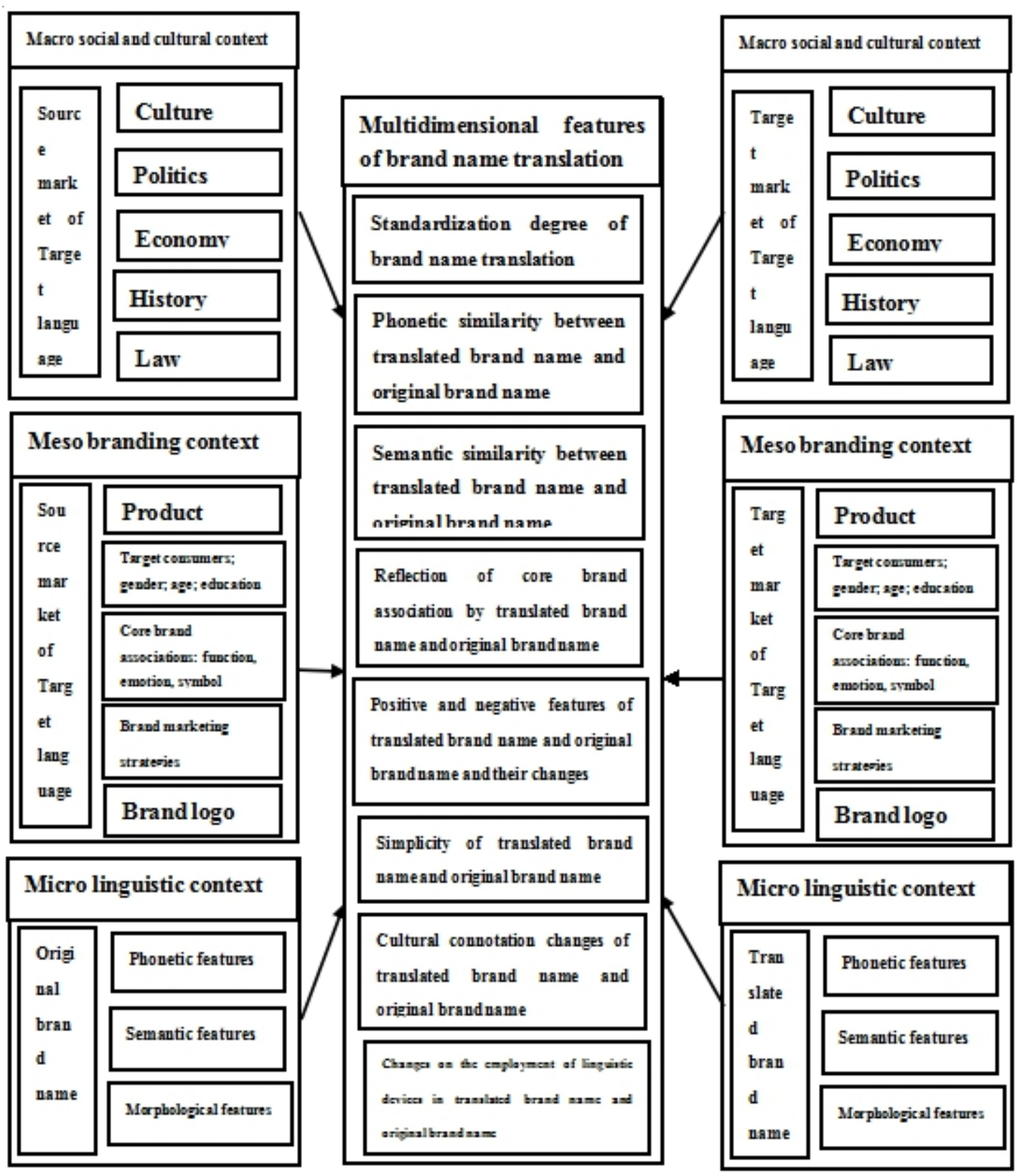

Figure 1. Generation and formulation frame of brand name translation's "meaning-senses"

\subsection{Brand Name Translation: Generation of "Meaning-senses"}

\subsubsection{Macro Contextual Factors in the Generation of Brand Name Translation's "meaning-senses"}

Culture is a multidimensional collection of knowledge, belief, art, morality, law and custom needed by a society (community). Only in specific social and cultural context can language have real meaning. The influence and restriction of macro cultural context on the interpretation of "meaning-senses" of brand name translation are primarily reflected in the legitimacy of cultural connotation expressed through word choices of a brand name translation. Every brand name's value is rooted in particular social and social background, and even contains particular cultural image or association of national psychology. When a brand containing specific cultural image attempts to enter into the target market of target language, it is necessary to make a cross-language and cross-cultural detection on its brand name translation, examining whether the brand name translation conflicts with politics, economy, culture, history and law of target market and offends relevant cultural taboos. If conflicts occur, it has to choose another brand name translation conforming to the culture of target market according to the dual contexts of both source language and target language, or it would result in failure of enterprise marketing 
and even cause political conflict between two countries. For instance, the name of "Prado", which is sold by Japanese Toyota in Chinese market, originates from Spanish. It conceptually means "(fashionable circles) walks" or (exclusive residential district) Park Avenue, having cultural connotation of "fashionable and high-end". Certain translator homophonically translated it into “霸道”, whose conceptual meaning is "occupy roads forcibly". The intended "meaning" of "霸道" probably is that its automobile can "dominate or reign on the Chinese roads". However, “霸道” marketed by Japanese arouses strong dissatisfaction among Chinese consumers and triggers the association of the historical fact that Japanese has invaded and bullied China. The brand name of “霸道” hurts severely the national pride of Chinese people and thus causes serious protest. For another instance, “联想”, a famous Chinese computer brand, used to apply "Legend" persistently as its English name regardless of the fact that "Legend" had been registered in European and American markets. Later it had to spend huge money on renaming itself into "Lenovo" when it implemented international brand strategy. In 2009, Microsoft released a research engine brand named Bing, whose pronunciation is the same as “病” (disease) in Chinese. In order to avoid possible cultural misunderstanding, Bing chose the word “必应”, which is close to English brand name in pronunciation, and matched the corresponding pinyin "biying". These examples demonstrate that brand name translation has to consider factors of original brand name that conflict with politics, economy, culture, history and law of target text language. Besides, it also has to adjust the "meaning" of cultural connotation of the translated brand name according to main relevant contextual factors of source language and target language.

\subsubsection{Meso Contextual Factors in the Generation of Brand Name Translations' "meaning-senses"}

Branding is the strict process of establishing brand awareness and capturing consumer's loyalty (Wheeler, 2014). Main factors of Meso branding context play a decisive role in the generation of brand name translation's "meaning-senses". Basing on product attributes, enterprises identify demands of target consumers, devise brand marketing strategies, position core brand associations, adopt corresponding brand logos and decide brand names and other branding measures to deliver information that meets functional, emotional and (or) symbolic demands of target consumers, then establishing brand awareness and capturing consumer's loyalty. Consequently, during brand name translation, translators have to understand enterprise's brand building activities comprehensively and conduct detailed investigation on product attributes, target consumers, brand marketing strategies, core brand associations and brand logo of enterprise in the markets of both source language and target language.

Generally, product attributes of a brand influence the choices of characters or pronunciation of its translated brand name, affecting the changes of positive and negative meanings in the translated brand name. For instance, $\mathrm{P} \& \mathrm{G}$, one of the largest household necessity companies in the world, holds many sub brands. Chinese translations of its brands mostly choose commendatory terms. On the one hand, they well coordinate with English brand names in meaning and pronunciation. On the other hand, they precisely reflect product characteristics, brand images and consumer positioning. Thus, these Chinese brand name translations not only arouse happy associations and enhance product affinity and trust among consumers, but also save expenditure in product marketing largely. For instance, Chinese translation of P\&G company itself is “宝洁”, in which “宝” means "superior quality of company's products and “洁” manifests that product and industry attribute of company is "daily chemical product category". Chinese translations of $P \& G$ ' sub brands primarily apply characters that can indicate product characteristics to transfer product effects of brands, such as "飞" and "咝" in the Chinese translation “海飞丝” of “Head \& Shoulders”, “飘” and “柔” in the Chinese translation “飘柔” of "Rejoice", which make people associate with the image of a young girl flowing hair and desire to buy them strongly. In the Chinese translation “帮宝适” of “Pampers”, “宝”(baby)) shows that target consumers of its product position in the "babies", and “帮” and “适” imply that "the function of its products is to make babies comfortable while using them". “汰” and “渍” in the Chinese translation “汰渍” of "Tide", a detergent brand, clearly convey that "this product has the function of removing obstinate stains ”. “奇乐”, Chinese translation of "Cheer", indicates the effects that its products can be suitable for water in different temperatures. Additionally, the translations of brand names that have entered into market earlier would influence the choices of latter ones of the same product category in translation strategies and methods. For instance, fast food brands, such as KFC (肯 德基), McDonald's (麦当劳), PizzaHut (必胜客) and Burger King(汉堡王), employ three-character words to designate product's origin and features of products or target consumers.

Consuming habits, language processing pattern, gender, age and education of target consumers have huge impacts on the generation of a translated brand name' "meaning", reflected in the phonetic, semantic and morphological changes of the translated brand name. As to language processing pattern, Li \& Shooshtari (2003) has observed in E-C brand name translation study that most Americans are keen on phonetic appeals of brand names while most of Chinese people understand the meanings of brand names through the ideographic 
characteristics of language. Therefore, when a brand enters into Chinese or American market, language processing habits of consumers have to be taken into consideration in translation. For instance, "Coca Cola", the most famous soft drink brand in the world, primarily considers sound appeal to target consumers as an English brand name. However, its Chinese translation “可口可乐” takes Chinese consumers' preference for meaning into consideration and, basing on its homophonic sounds with English brand name, adds core brand associative meaning (that is the naming meaning of brand) “美味可口, 开启快乐” (delicious and tasty, opening happiness). Equally, gender, age and education of target consumers also affect the generation of a translated brand name's "naming meaning". For instance, female cosmetic brand names are usually translated with specific lexicon to indicate gender attribute to promote purchases. For example, as a personal name, normally Estee Lauder is translated into “艾斯蒂.劳德”; as a cosmetic brand name, it is translated into “雅诗兰黛”. For more examples, "Clinique", a sub brand of Estee Lauder, means "clinic" in English while its Chinese translation is “倩 碧”(beautiful countenance and green jade). Guerlain, a skincare brand starting business with perfumes, is the last name of its founder. And it chooses “娇兰” as its Chinese translation.

The influence of brand marketing strategy on brand name translation is chiefly reflected in standardization degree of translated brand name, phonetic and semantic similarity degree between original brand name and translated brand name, and succinctness of translated brand name. Standardization and localization is a continuity of marketing activities. Standardization of brand name means scale economy brought by saving cost from promotion, distribution and packaging, while localization of brand name increases cost. If an enterprise attempts to implement standardization (or globalization) strategy in the target market of target language, the standardization degree of its brand name needs to be increased. The most ideal state is the application of "zero translation", that is maintaining the original brand name, such as IBM. If it does need a brand name translation but demands for pronunciation and meaning can not be satisfied at same time, then it inclines to keep the phonetic similarity between the translated brand name and original brand name, which can help achieve global advertising campaign easily and construct a consistent global brand image. For instance, "LEXUS" was an automobile brand popular in Chinese market in 1990s. Its Chinese translation “凌志” accorded with the characteristics of target consumers and catered for Chinese cultural consumption psychology at that time, but it was abandoned by Toyota in 21st century and changed into a new transliterated name “雷克萨斯”. Similarly, another Toyota automobile brand Corolla, whose previous Chinese translation was “花冠”, was retranslated into “卡罗拉”. Although both “雷克萨斯” and “卡罗拉” are meaningless as transliterated names, they are closer to original English brand names in pronunciation. Additionally, they fit Toyota global standardization strategy and more match other elements (like brand image and brand communication) of company brand construction. They are also more helpful to produce consistent cognition towards brand among potential target consumers and to reduce advertising cost. If a company attempts to implement localization strategy in target market of target language, the standardization degree of translated brand name is lower. The naming meaning of translated brand name would differ greater from that of original brand name. For instance, City, a sub automobile brand of Guangzhou Honda, emphasized Chinese translation's phonetic similarity with original English brand name in early stage and was translated into “思迪” whose meaning is absent. Later, with further implementation of localization strategy, City was retranslated into “锋范”, in which “锋” stands for challenge spirit of being remarkable and striving forward and “范” represents that its target consumers are leaders and examples of young talents. “锋范” differs significantly from original English brand name either in pronunciation or meaning, but it complies with company's marketing strategy of brand localization and accomplishes seamless connection with company's various marketing activities. In addition, as a short brand name is easier to be remembered (Robertson, 1989), the length of a brand name translation is usually adjusted according to linguistic features of target language. For example, Hush Puppies, a leisure brand, is translated into “暇步士” with renaming method according to norms of Chinese language, highlighting brand name's naming meaning "comfort and leisure" and successfully avoiding possible excessive syllables caused by pure transliteration.

Brand association reflects positioning of brand marketing strategy. The potential value of brand names often depends on concrete brand associations. Therefore, core brand associations are decisive factors in deciding the "meaning-senses" of a translated brand name, and affect semantic similarity of translated brand name with original brand name, phonetic changes and adoption of linguistic devices. Generally, besides directly employing popular words capable of presenting core brand themes or associations (for instance, "Origin", a famous skincare brand of Estee Lauder, is translated into Chinese “悦木之源”, which directly emphasizes pure natural plant formula), an enterprise can rename a brand with proper names such as place names or personal names and invented words. Adoption of the name or last name of an enterprise's founder to name a brand is to identify who founds or owns the enterprise, what kind of products or services it sells, where to find these products, or what market it covers (Rivkin \& Sutherland, 2004). Then quality of the brand can be identified. In this sense, the 
naming meaning of this brand name is "core brand associative meaning" endowed by branding, different form its literal meaning. Therefore, the meaning of translated brand name will depend on the transmission of brand naming meaning rather than the transference of literal meaning. If the transliterated name of brand's founder can deliver brand naming meaning, then the brand can be transliterated, such as “蒂芙尼”, Chinese translation of "Tiffany", a famous jewelry brand. If the transliterated name of brand's founder can not deliver the naming meaning a brand needs to express, the brand needs to adjust its brand name types and employs other forms as its translation. BENZ, one of world's top ten automobile companies, is the last name of its founder as an English name. However, its Chinese translation does not keep personal name and adopts a verb to convey the brand's naming meaning "top quality, top performance and top grade". Johnson \& Johnson, a famous company of medical health care and consumer care products in the world, is also the last name of its founder as an English brand name, but its Chinese translation does not employ transliteration to keep original personal name as well. It chooses “强生” fit for Chinese consumers' preference as its brand name translation to deliver its naming meaning which represents its function and core emotional associations. McDonald's, a large multinational chain restaurant globally, originates from its founder's name as English brand name. However, when it is translated, not only brand name type is changed, but also the meaning of its translation is very different from that of English brand name. Chinese brands named after personal names lack this kind of flexible strategy in translation and are usually translated with pinyin. For instance, Chinese brand “李宁” is translated into “Li Ning” in English. Since “李宁” is just a famous Chinese gymnastic Olympic champion as a personal name, the background and specialty of “李宁” brand can be hardly understood by potential foreign consumers. Consequently, the naming meaning produced by associations of Chinese brand name is lost in the translated name in pinyin. The adoption of invented words in a brand name is to present critical attribute or characteristic of products through combination of roots. For instance, GEOX, the top Italian leisure brand, is an invented word generated from Greek "GEO", which means the ground under foot. With unknown number " $\mathrm{X}$ " as technique symbol, it associates product attributes of its shoes with high technique, indicating product quality. Differently, its Chinese translation “健乐 士” leads to target consumers and indicates information of product equality through walking experience.

Brand logo is the part of a brand that can be recognized and easily to be remembered but can not be addressed linguistically, including symbol, picture or obvious color or typeface. Brand logo and brand name together construct a complete brand concept. Therefore, generally brand logo would affect the choice of translated brand name's "meaning" in a brand name translation. For instance, the picture in brand logo of Crown, a Japanese Toyota automobile brand, is a crown. The picture of crown basically has the same meaning in the cognition of people of different nations in the world. Originally it means "the hat worn by a king or queen and symbolizing absolute power", symbolizing great honor and luxury. Therefore, when it seeks for a Chinese translation, its brand name translation is hard to adopt transliteration strategy to keep phonetic similarity with English brand name, resulting in the choice of directly translated name “皇冠”. There are many more examples choosing consistency of brand logos to brand names, such as famous computer brand Apple, whose brand logo is an apple with one bite, resulting in the direct adoption of “苹果” in its Chinese translation.

\subsubsection{Micro Contextual Factors in the Generation of Brand Name Translation's "meaning-senses"}

Brand name exists in the form of linguistic symbol. Phonetic, semantic and morphological characteristics of brand name influence the expression of brand name's meaning. Therefore, pronunciation, semantic meaning and morphological form as micro linguistic contextual factors become the core of translators' focuses.

Pronunciation is an important component of language, having partly arbitrary and partly natural relation with meaning. People naming brands often use physical expressions such as pronunciation and metres to evoke people's associations between brand names and products. For instance, Coca Cola makes full use of alliteration to present symbolic sense of "harmonious beauty" and “music" and its Chinese translation “可口可乐” also displays the same symbolic sense with the same method.

Meaning is the core component of language, and can be divided into conceptual meaning, connotative meaning, social meaning, affective meaning, reflected meaning, collective meaning and thematic meaning (Leech, 1974). Among them, conceptual meaning is the core factor of linguistic communication. Connotative meaning, social meaning, affective meaning, reflected meaning and collective meaning are collectively referred as associative meaning. And thematic meaning is the meaning attached by special organized way of information content. Combining semantic characteristics and forming rules of brand naming, Zhu (2003), divided semantic status of brand names into denotative meaning, symbolic meaning and named meaning on the basis of Leech's semantic classification. Denotative meaning is the first semantic status of brand naming, the correlative meaning formed by brand naming symbols and their denoted objects, and the basic meaning of brand naming. In this paper, it is called "designative meaning". Symbolic meaning is the literal meaning in brand naming meaning and a part of 
conceptual meaning classified by Leech. It is called "literal meaning" in this paper. Named meaning is the meaning acquired through associative mode, the deep status of brand naming meaning established on the basis of symbolic meaning and denotative meaning, and the most important semantic content pursued by persons naming brands and formed in the process of brand naming symbolization and denotation. It is also referred as "named meaning" in this paper. For instance, the named meaning of Hush Puppies (leisure shoes) does not lie in the designative relation between naming symbols and designated products but the re-connection between symbolic meaning and shoes. The naming meaning "comfort and leisure", which is generated by symbolic meaning and shoes jointly, is the genuine meaning of the brand name. Its Chinese translation “㗇步士” works in the same way.

Morphological form is the expression form of language, determining linguistic meaning along with pronunciation and semantic meaning. As short brand name is easier to be memorized, the morphological form of a translated brand name generally is required to be short and simple, which is measured by the number of words, English syllables or strokes in Chinese characters composing brand name's length (Robertson, 1989). For instance, in contemporary Chinese, since disyllabic and three-syllable words are easier to pronounce, spread, understand and memorize, Chinese naming inclines to apply disyllabic and three-syllable words (Huang \& Chan, 2002). Therefore, Chinese translations of foreign brand names mostly are disyllabic and three-syllabic, such as ““德芙(Dove)”, “宜家(Ikea)” and “惠而浦(Whirlpool)”. And those adopting four syllables largely take morphological forms and naming meanings of original brand names into consideration, such as“劳斯莱斯(Rolls Royce)” and “雅诗兰黛(Estee Lauder)”.

\subsection{Brand Name Translation: Formulation of "Meaning-senses" (See Figure 2)}

According to above analysis, the generation and formulation of a brand name translation's "meaning-senses" is influenced jointly by main macro, meso and micro contextual factors of both source language and target language. Contextual factors of macro culture primarily affect the appropriateness of a brand name translation's "cultural meaning" and legitimacy of the brand name, and then influence acceptance of target consumers toward the brand. Contextual factors of meso branding chiefly influence the determination on the "named meaning" of a brand name translation, and then affect target consumers' perception of functional, emotional and (or) symbolic (self-fulfillment) interests. Contextual factors at micro linguistic level directly influence the proper expression of a brand name translation's "named meaning", and then affect target consumers' understanding of a brand name translation's "meaning-senses". Therefore, brand name translation should be guided by the "cultural meaning" ascertained by main contextual factors conforming to macro culture, adopt proper "designative meaning" and "literal meaning" determined by contextual factors at micro linguistic level, and deliver translated brand name' "named meaning" determined by contextual factors at meso branding level.

Taking “Estee Lauder (雅诗兰黛)", a top cosmetic brand in the world, mentioned earlier as an example, the following section will discuss the generation and formulation of "meaning-senses" in the process of brand name translation. Estee Lauder, established in 1946 in New York, USA, is a cosmetic brand named after Lady Estee Lauder, its founder. Estee Lauder, chiefly targeted at female consumers, intends to make every female beautiful. Now it has become a flagship brand of American Estee Lauder Companies, famous for its anti-aging and repair skincare (Table 1).

According to Table 1, we can find that Estee Lauder, a brand established in United State, insists on the use of Chinese pronunciation, semantic meaning and morphological form, which fit for Chinese macro social and cultural context, to express naming meaning of "elegance, beauty and fashion" determined by Estee Lauder's meso branding context when it enters into Chinese market. Firstly, Estee Lauder, insisting on using brand strategy of standardization, attempts to convey the same brand interests and meet target consumers' demands for "elegance, beauty and fashion" in different markets, through the same brand naming meaning, to establish a unified brand image. Secondly, as Estee Lauder is the name of its founder and has experienced years of brand communication activities closely related to its brand naming meaning in United States, the name itself has long been connected with its brand naming meaning in United States. When it enters into China, it would be translated into “艾斯蒂.劳德” according to pronunciation rules of English personal names. “艾斯蒂.劳德” only has the significance of designating a foreign personal name and has no association with product attributes and functions. The naming meaning of the brand would disappear completely. On the contrary, Chinese translation “雅诗兰黛” not only formulates relative meaning with its designated object "skincare", representing "skincare", but also conveys rich information relevant to brand products. On the one hand, the combination of Chinese characters, different from conventional ones, transmits products' information that it origins from "west" and draws support from Chinese consumers' cultural stereotype image of western product, having "superior quality". On the other hand, every character has rich connotative meaning respectively. According to Xinhua Dictionary, 
“雅” means “happiness, grace and elegance”. “诗” is a literary genre, reflecting real life and expressing feelings through language with rhythm and rhymes. It is frequently used to describe beautiful things which are full of living delights or can trigger strong feelings in people. "兰” refers to "orchard", which metaphorically describe “fragrance and elegance". It is often used in females' names. “黛” originally means "bluish black pigment" and extends to means "beautiful women", being a fine image containing positive meaning in Chinese as well. Choosing these four Chinese characters as its Chinese brand name translation has taken letters S, L and D in the original brand name Estee Lauder and morphological forms into consideration, achieving phonetic similarity and consistency of morphological simplicity to certain extent. The Chinese translation indicates the users of products, transmits product functions and expresses brand naming meaning "elegance, beauty and fashion". When the designative meaning of Chinese translation “雅诗兰黛” associates with its literal meaning, it evokes its brand named meaning "elegance, beauty and fashion".

Table 1. Contexts of source and target languages translation of estee lauder relies on and their main factors

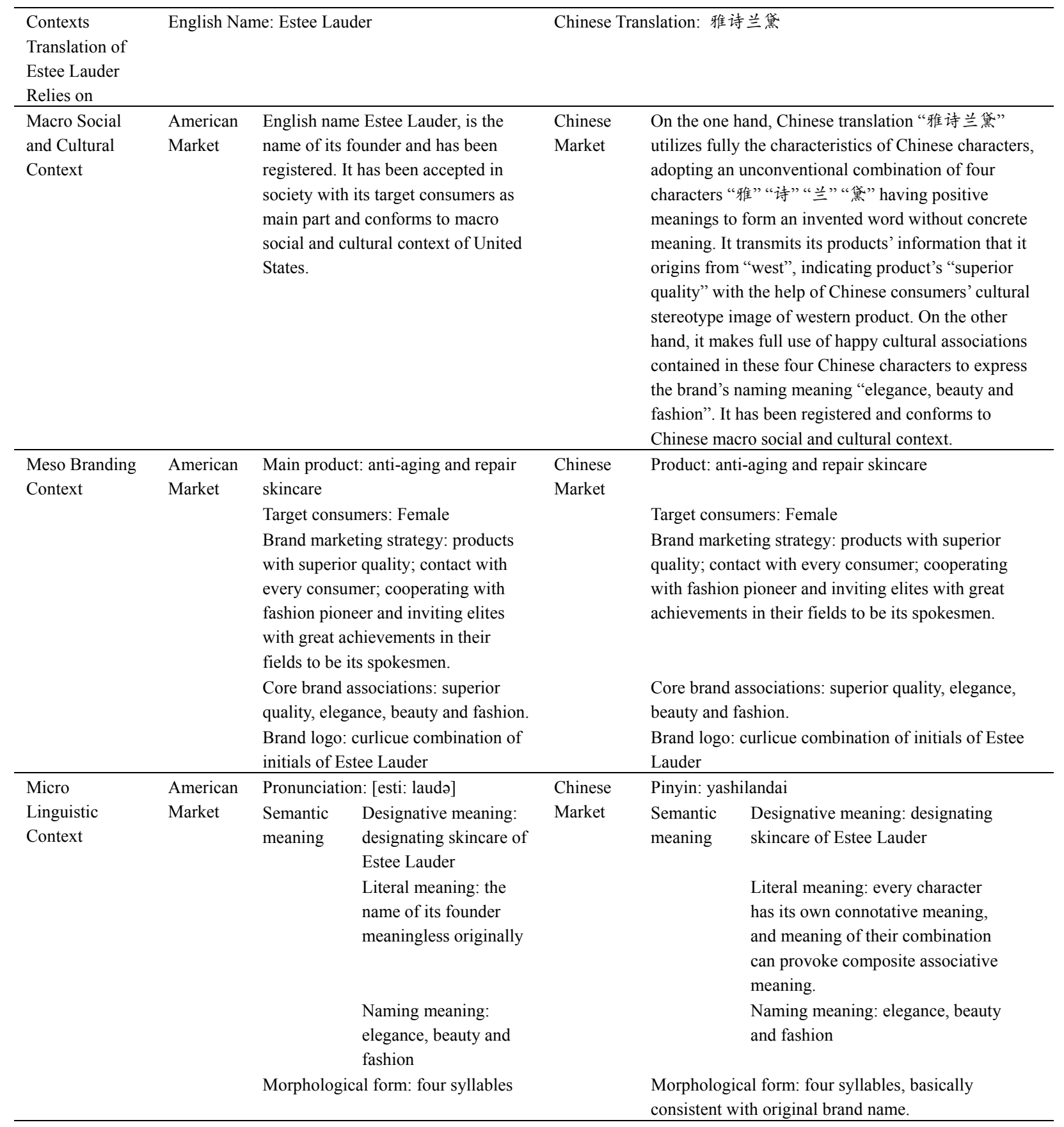




\section{Conclusions}

From the macro, meso and micro contexts brand name translation relies on, this research discusses the generation mechanism and formulation principles of "meaning-senses" in the process of brand name translation with specific examples of E-C brand name translation. This research observes that the generation and formulation of a brand name's "meaning-senses" is affected and constrained by main factors of macro, meso and micro contexts a brand name relies on. Political, economic, cultural, historical and legal factors at macro cultural level influence the cultural connotation and legitimacy of brand name translations and then impacts on the choice of cultural meaning of brand name translations. Product, target consumers, brand marketing strategy, core brand associations and brand logo at meso branding level affect standardization degree, phonetic and semantic consistency, morphological simplicity, presentations of brand association and changes of positive and negative elements, and then influence the determination on brand named meanings. Phonetic, morphological and semantic elements at micro linguistic level, working together, influence phonetic and semantic (designative meaning and literal meaning) changes of brand name translations and employment of linguistic devices, and then affects the expressions of "brand named meaning" of brand name translations. Therefore, the meaning of brand name translations should not be confined to micro translation units (original meaning of words or phrases). Instead, brand name translation should be brought into macro cultural context and meso branding context and adhere to micro linguistic contextual factors meeting demands of macro cultural context, delivering the "named meaning" identified by meso branding contextual factors.

\section{Acknowledgement}

This paper is supported by the Fundamental Research Funds for the Central Universities, Southwest Minzu University (Grant No. 2015SZYQN131).

\section{References}

Dong, L. C., \& Helms, M. M. (2001). Brand name translation model: A case analysis of US brands in China. Brand Management, 9(2), 99-115. https://doi.org/10.1057/palgrave.bm.2540058

Eggins, S. (1994). An Introduction to Systematic Functional Linguistics. London: Pinter.

Francis, J., Lam, J., \& Walls, J. (2002). The impact of linguistic differences on international brand name standardization: A comparison of English and Chinese brand names of Fortune-500 companies. Journal of International Marketing, 10(1), 98-116. https://doi.org/10.1509/jimk.10.1.98.19528

Gardner, B. B., \& Sidney, J. L. (1955). The Product and the Brand. Harvard Business Review, (March- April) 33-39.

He, Z. R. (1992). Pragmatics and CE/EC translation. Foreign Language Education, (1), 21-25.

Huang, Y. Y., \& Chan, A. K. K (2002). The principle and special features in Chinese Branding. Nankai Business Review, (1), 68-71.

Li, F., \& Shooshtari, N. H. (2003). Brand naming in China: Sociolinguistic implications. The Multinational Business Review, 11(3), 3-21. https://doi.org/10.1108/1525383X200300014

Li, Z., \& Murray, L.W. (2002). Naming products in China: Local or foreign branding. Journal of Asia-Pacific Business, 3(3), 53-71. https://doi.org/10.1300/J098v03n03_04

Rivkin, S., \& Sutherland, F. (2004). The making of a name: The inside story of the brands we buy. Oxford: Oxford University Press.

Robertson, K. (1989). Strategically desirable brand name characteristics. Journal of Consumer Marketing, 6, 61-70. https://doi.org/10.1108/EUM0000000002563

Schmitt, B., \& Zhang, S. (2012). Selecting the right brand name: An examination of tacit and explicit linguistic knowledge in name translations. Journal of Brand Management, 19(8), 655-665. https://doi.org/10.1057/bm.2011.62

Shaw, D. (1987). The translation context: Cultural factors in translation. Translation Review, (23), 25-29. https://doi.org/10.1080/07374836.1987.10523398

Stern, W. (1983). A good name could mean a brand of fame. Advertising Age, 54(3), M53-54.

Usunier, J. C., \& Shaner, J. (2002). Using linguistics for creating better international brand names. Journal of Marketing Communications, (8), 211-228. https://doi.org/10.1080/13527260210146000

Verschueren, J. (1999). Understanding Pragmatics. London: Edward Arnold (Publishers) Limited. 
Wang, K. Y. (2008). Translation as Process and as Product: A Perspective of Cognition, Language Communication and Meaning. Beijing: Chinese Social Sciences Press.

Wheeler, A. (2014). Designing Brand Identity. Beijing: Publishing House of Electronics Industry.

Zhang, S., \& Schmitt, B. H. (2001). Creating local brands in multilingual international markets. Journal of Marketing Research, 38(2), 313-325. https://doi.org/10.1509/jmkr.38.3.313.18869

Zhang, S., \& Schmitt, B. H. (2004). Activating sound and meaning: The role of language proficiency in bilingual consumer environments. Journal of Consumer Research, 31(June), 220-228. https://doi.org/10.1086/383437

Zhu, Y. J. (2011). Study on Trademark naming (shangbiao mingming yanjiu) [M]. Shanghai: Shanghai Foreign Language Education Press.

\section{Copyrights}

Copyright for this article is retained by the author, with first publication rights granted to the journal.

This is an open-access article distributed under the terms and conditions of the Creative Commons Attribution license (http://creativecommons.org/licenses/by/4.0/). 